\title{
Has lung ultrasound an impact on the management of patients with acute dyspnea in the emergency department?
}

\author{
Alberto Goffi ${ }^{1,2^{*}}$, Emanuele Pivetta ${ }^{3,4}$, Enrico Lupia ${ }^{5,6}$, Giulio Porrino ${ }^{1,5}$, Marina Civita ${ }^{1}$, Emanuela Laurita ${ }^{1}$, \\ Giulietta Griot ${ }^{1}$, Giovanna Casoli ${ }^{1,7}$ and Gian Alfonso Cibinel $^{1}$
}

Lung ultrasound (LUS) has emerged as a rapid, immediately available, non-invasive bedside tool for detection of several pulmonary and/or pleural diseases [1,2]. However, no data are available for its impact on patient management in the emergency department.

We studied 50 adult patients (25 women, median age 80.5 years, interquartile range 12.3 years) presenting with acute undifferentiated dyspnea to the emergency department of 'Edoardo Agnelli' Hospital (Pinerolo, Turin), and evaluated the LUS diagnostic impact by comparing the main diagnosis (cardiac, respiratory, or combined dyspnea), the most likely pathophysiologic dysfunction, and the etiological diagnosis, as indicated by the emergency physician caring for the patient, before and after LUS. Reference diagnosis was established by two expert emergency physicians, blinded to LUS results, who independently reviewed the entire medical record. Moreover, we asked the emergency physician how LUS findings changed patient management.

We used a previously described eight-region scanning protocol [3] and assessed five major syndromes (see Figure 1) [1]. Diffuse interstitial syndrome (IS) was the most common finding (58\%), followed by pleural effusion (52\%), focal IS (18\%), alveolar consolidation (14\%), and pneumothorax (8\%). Twenty-seven patients had two or more LUS pathological findings (for example,
17 patients showed pleural effusion and IS), while in six patients LUS detected no alterations.

The agreement between clinical assessment and reference diagnoses was fair (Cohen's kappa coefficient = $0.25,0.32$, and 0.26 for main, pathophysiologic, and etiological diagnosis, respectively; $P<0.01$ ), while agreement was excellent between LUS-implemented and reference diagnoses $($ kappa coefficient $=0.94,0.84$, and 0.81 , respectively; $P<0.01)$. Overall, LUS changed the main clinical diagnosis in $44 \%$ of cases. The agreement for the different main diagnosis is reported in Figure 2.

Therapeutic management was changed, because of LUS findings, in $58 \%$ of patients. LUS led to prescribing a new drug in 19 cases, and to holding a drug previously considered for treatment in 10 cases; moreover, LUS results led to a new procedure (for example, thoracentesis) being performed in six cases, and to a change of the disposition plan in five cases.

Our study is the first to address the issue of how LUS affects patient management in real-world practice. We found a high diagnostic and therapeutic impact, mainly related to the sequential approach used integrating clinical assessment and LUS, that paired the detection of IS, a very sensitive but poorly specific ultrasound sign [1], with the pretest probability of clinical assessment. These data need to be confirmed in larger cohort and multicenter studies.

\footnotetext{
*Correspondence: alberto.goffi@gmail.com

'Department of Emergency Medicine, ASL TO3, Ospedale Civile "E. Agnelli", Via Brigata Cagliari 39, 10064, Turin, Pinerolo, Italy

${ }^{2}$ Department of Critical Care Medicine, St Michael's Hospital, 30 Bond Street, Toronto, ON M5B 1W8, Canada

Full list of author information is available at the end of the article
} 

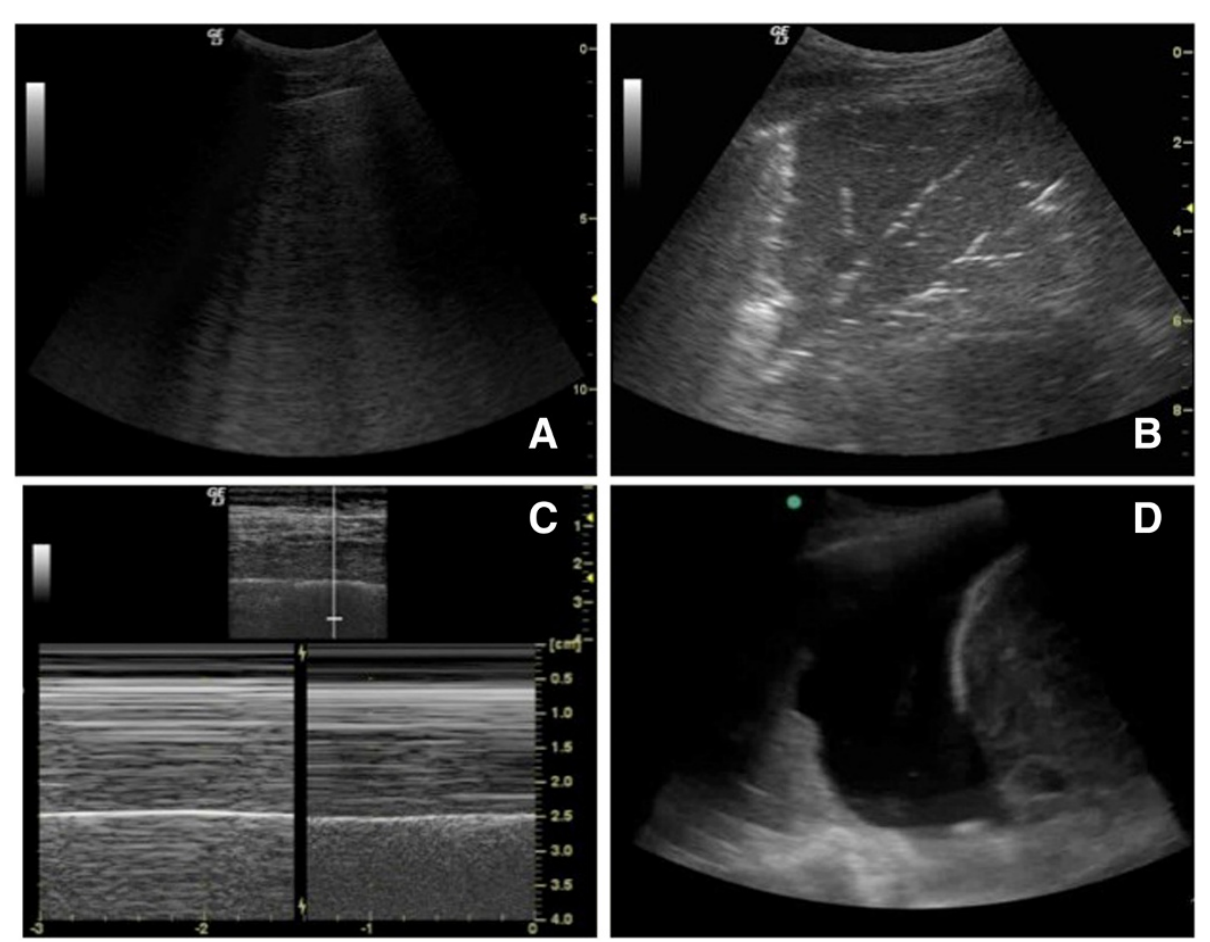

Figure 1 Lung ultrasound major syndromes. (A) Diffuse B-lines representing interstitial syndrome. (B) Alveolar consolidation. (C) M-mode findings of a pneumothorax (stratosphere sign or bar code sign; left), and a normal lung (seashore sign). (D) Pleural effusion.

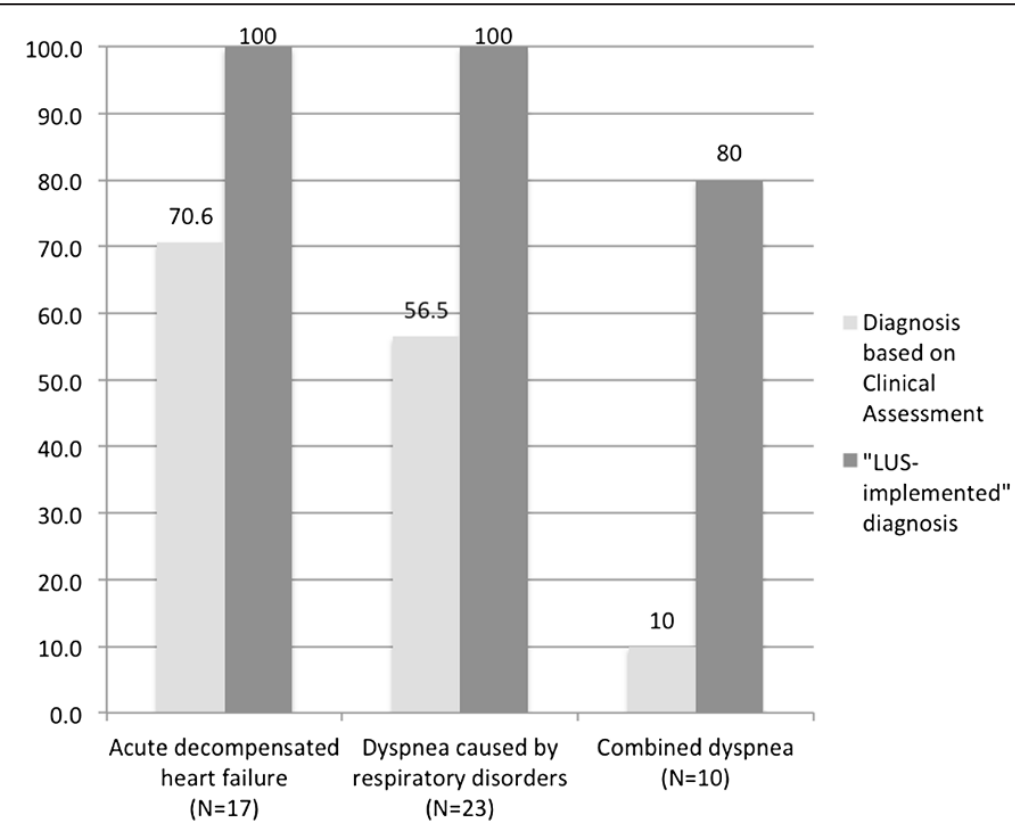

Figure 2 Agreement for the main diagnosis between clinical assessment or lung ultrasound (LUS)-implemented assessment, and reference diagnosis. 


\section{Abbreviations}

IS: Interstitial syndrome; LUS: Lung ultrasound.

\section{Competing interests}

The authors declare that they have no competing interests.

\section{Acknowledgments}

Informed consent for participation and for publication was obtained from all patients before inclusion.

\section{Author details}

'Department of Emergency Medicine, ASL TO3, Ospedale Civile "E. Agnelli", Via Brigata Cagliari 39, 10064, Turin, Pinerolo, Italy. ${ }^{2}$ Department of Critical Care Medicine, St Michael's Hospital, 30 Bond Street, Toronto, ON M5B 1W8, Canada. ${ }^{3}$ Cancer Epidemiology Unit, CPO Piemonte and CeRMS, University of Turin, AO Città della Salute e della Scienza, Via Santena 7, 10126, Turin, Italy. ${ }^{4}$ Department of Emergency Medicine, Division of Emergency Ultrasound, Brigham and Women's Hospital, Boston, Neville House, 75 Francis Street, 02115, Boston, MA, USA. ${ }^{5}$ Department of Emergency Medicine, AO Città della Salute e della Scienza, Presidio San Giovanni Battista - Molinette, Corso Bramante 88, 10126, Turin, Italy. ${ }^{6}$ Department of Medical Sciences, University of Turin, Via Genova 3, 10126, Turin, Italy. ${ }^{7}$ Department of Emergency Medicine, ASL TO1, Martini Hospital, Via Tofane 71, 10141, Turin, Italy.

\section{Published: 27 August 2013}

\section{References}

1. Volpicelli G, Elbarbary M, Blaivas M, Lichtenstein DA, Mathis G, Kirkpatrick AW, Melniker L, Gargani L, Noble VE, Via G, Dean A, Tsung JW, Soldati G, Copetti R, Bouhemad B, Reissig A, Agricola E, Rouby JJ, Arbelot C, Liteplo A, Sargsyan A, Silva F, Hoppmann R, Breitkreutz R, Seibel A, Neri L, Storti E, Petrovic T, International Liaison Committee on Lung Ultrasound (ILC-LUS) for International Consensus Conference on Lung Ultrasound (ICC-LUS): International evidence-based recommendations for point-of-care lung ultrasound. Intensive Care Med 2012, 38:577-591.

2. Lichtenstein DA, Mezière GA: Relevance of lung ultrasound in the diagnosis of acute respiratory failure: the BLUE protocol. Chest 2008, 134:117-125.

3. Cibinel GA, Casoli G, Elia F, Padoan M, Pivetta E, Lupia E, Goffi A: Diagnostic accuracy and reproducibility of pleural and lung ultrasound in discriminating cardiogenic causes of acute dyspnea in the emergency department. Intern Emerg Med 2012, 7:65-70.

doi:10.1186/1364-8535-17-R180

Cite this article as: Goffi et al:: Has lung ultrasound an impact on the management of patients with acute dyspnea in the emergency department? Critical Care 2013 17:R180. 